\title{
Recurrent Bleeding in Hemorrhagic Moyamoya Disease : Prognostic Implications of the Perfusion Status
}

\author{
Kyung-II Jo, M.D., Min Soo Kim, M.D., Je Young Yeon, M.D., Jong-Soo Kim, M.D., Ph.D., Seung-Chyul Hong, M.D., Ph.D. \\ Department of Neurosurgery, Samsung Medical Center, Sungkyunkwan University School of Medicine, Seoul, Korea
}

\begin{abstract}
Objective : Hemorrhagic moyamoya disease (hMMD) is associated with a poor clinical course. Furthermore, poorer clinical outcomes occur in cases of recurrent bleeding. However, the effect of hemodynamic insufficiency on rebleeding risk has not been investigated yet. This study evaluated the prognostic implications of the perfusion status during the clinical course of adult hMMD.

Methods : This retrospective study enrolled 52 adult hMMD patients between April 1995 and October 2010 from a single institute. Demographic data, clinical and radiologic characteristics, including hemodynamic status using single photon emission computed tomography (SPECT), and follow up data were obtained via a retrospective review of medical charts and imaging. Statistical analyses were performed to explore potential prognostic factors

Results : Hemodynamic abnormality was identified in 44 (84.6\%) patients. Subsequent revascularization surgery was performed in 22 (42.3\%) patients. During a 58-month (median, range 3-160) follow-up assessment period, 17 showed subsequent stroke (hemorrhagic $n=12$, ischemic $n=5$, Actuarial stroke rate 5.8 $\pm 1.4 \%$ /year). Recurrent hemorrhage was associated with decreased basal perfusion ( $\mathrm{HR} 19.872 ; 95 \% \mathrm{Cl}=1.196-$ 294.117) and omission of revascularization (10.218; 95\%; $\mathrm{Cl}=1.532-68.136)$.

Conclusion : Decreased basal perfusion seems to be associated with recurrent bleeding. Revascularization might prevent recurrent stroke in hMMD by rectifying the perfusion abnormality. A larger-sized, controlled study is required to address this issue.
\end{abstract}

Key Words : Cerebral hemorrhages · Moyamoya disease $\cdot$ Cerebral revascularization $\cdot$ Hemodynamics.

\section{INTRODUCTION}

Moyamoya disease (MMD) is a rare, chronic, and progressive cerebrovascular disorder characterized by stenosis and occlusion of the distal carotid and proximal middle and anterior cerebral arteries. MMD is also accompanied by the development of small collateral vessel networks at the base of the brain ${ }^{17)}$. Ischemia is the most common sign of this disease, and surgical revascularization seems to be helpful for this MMD patient subgroup $^{1,3,16)}$.

The hemorrhagic subtype is found in 20\% of MMD patients ${ }^{1)}$. This subtype is associated with a poor clinical course compared with other MMD subtypes ${ }^{15,19)}$. Only half of patients experience adequate recovery after the first hemorrhagic event ${ }^{7)}$. Furthermore, poorer clinical outcomes can be expected after recurrent bleeding ${ }^{19)}$. Currently, limited data is available regarding the clinical course of hemorrhagic MMD (hMMD) ${ }^{14)}$. Additionally, no definitive treatment method has been recognized as effective for preventing recurrent bleeding. Until recently, risk factors for recurrent bleeding were poorly understood. In a few studies, microbleeding observed on magnetic resonance imaging (MRI) or angiographic scans indicated the existence of dilated anterior choroidal and posterior communicating arteries deemed as hMMD predictors ${ }^{6,11}$. To the best of our knowledge, and despite hemodynamic insufficiency being the main pathophysiologic MMD finding, there have been no studies examining the effect of hemodynamic insufficiency on rebleeding risk in hMMD. Furthermore, even though a hemodynamic abnormality is a key pathologic feature of $\mathrm{MMD}$, ischemic events are poorly recognized $^{12)}$

Further verification regarding an hMMD clinical course and the role of hemodynamic insufficiency and other parameters on recurrent stroke is necessary. The current study reviewed the clinical course of adult patients with hMMD who were treated at our sponsoring institution. The goal of the present study was to rigorously clarify the clinical course and identify potential risk

- Received : June 12, 2015 • Revised : October 8, 2015 • Accepted : October 19, 2015

- Address for reprints : Seung-Chyul Hong, M.D., Ph.D.

Department of Neurosurgery, Samsung Medical Center, Sungkyunkwan University School of Medicine, 81 Irwon-ro, Gangnam-gu, Seoul 06351, Korea

Tel : +82-2-3410-3493, Fax : +82-2-3410-0048, E-mail : nsschong@skku.edu

- This is an Open Access article distributed under the terms of the Creative Commons Attribution Non-Commercial License (http://creativecommons.org/licenses/by-nc/3.0) which permits unrestricted non-commercial use, distribution, and reproduction in any medium, provided the original work is properly cited. 
factors for recurrent stroke in hMMD.

\section{MATERIALS AND METHODS}

\section{Patient selection}

This study included adult patients who were diagnosed with hMMD at our hospital between April 1995 and October 2010. This study included adult ( $\geq 18$ years) hMMD cases, which were defined as patients with a moyamoya phenomenon and who had initially presented with a hemorrhagic event. Moyamoya phenomenon was defined as unilateral or bilateral angiographic identification of severe stenosis showing an occlusion of the distal internal carotid or proximal middle and anterior cerebral arteries with prominent basal collaterals ${ }^{1}$. Hemorrhagic events included subarachnoid hemorrhage, intraparenchymal hemorrhage, and intraventricular hemorrhage. We excluded patients who did not undergo detailed angiographic evaluation with digital subtraction angiography (DSA) or a perfusion study using single positron emission computed tomography (SPECT). Early stage (Suzuki stage 1-2) unilateral MMD was excluded to minimize diagnostic ambiguities.

Between April 1995 and October 2010, 108 (male/female=25/83) adult moyamoya patients visited our hospital due to a hemorrhagic episode. Among these patients, 52 fulfilled the inclusion criteria. Exclusionary criteria were no assessable data from a DSA and/or SPECT ( $n=50)$, early stage unilateral MMD $(n=4)$, and inaccessible imaging and/or clinical data $(n=2)$.

SPECT was usually performed after resolution of the hematoma to minimize the mass effect of the hematoma on cerebral hemodynamics. The median time for the SPECT study after a hemorrhagic event was 1 month [interquartile range (IQR) 1-6]. All SPECT studies were performed before performing revascularization surgery. Impaired hemodynamics, defined as inadequate perfusion, was shown in the basal and/or acetazolamide brain SPECT. Decreased basal perfusion was defined as decreased perfusion on the basal SPECT. Impaired vascular reserve was defined as decreased perfusion during SPECT using acetazolamide. Basal and acetazolamide brain SPECT scanning to evaluate basal perfusion and vascular reserve were performed with a $99 \mathrm{mTc}-$ HMPAO, and the SPECT images were interpreted qualitatively by two experienced nuclear medicine physicians. The DSA was interpreted by two board-certified radiologists. Generally, we did not perform baseline studies, such as DSA and SPECT, on the patients with poor outcomes [>modified Rankin Scale (mRS) 4] after their initial presentation.

\section{Retrospective chart review}

The patients' demographic data, radiological findings, medical and surgical treatment, and outcomes were precisely analyzed through medical record reviews. The event-free survival rates were calculated in the two categories. Categories were recurrent bleeding and recurrent stroke, which included hemorrhagic and ischemic events. Ischemic events were included in the ischemic stroke and transient ischemic attack groups. Ischemic stroke was defined as the acute onset of a focal neurological deficit lasting $\geq 24$ hours and/or a high signal on diffusion weighted imaging (DWI). Transient ischemic attack (TIA) was defined as the onset of a focal neurologic deficit lasting $<24$ hours without signal change on DWI. A diagnosis of a hemorrhagic event was made on the basis of computed tomography or MRI.

\section{Statistical analyses}

Event-free survival was estimated with the Kaplan-Meier method.

Regarding age, gender, hypertension, smoking, antiplatelet use, perfusion status and surgical treatment, univariate analysis was performed using the log-rank test to evaluate statistical significance between each group

Multivariate analysis using a Cox regression test was performed to explore potential risk factors for rebleeding. The data were analyzed using SPSS version 19.0 (SPSS, Chicago, IL, USA).

\section{RESULTS}

Five of 52 patients were categorized as unilateral MMD. The

Table 1. Baseline patient characteristics

\begin{tabular}{lc}
\hline \multicolumn{1}{c}{ Characteristics } & Value \\
\hline Gender (male/female) & $8 / 44$ \\
Age, median (range) & 36 years $(21-60)$ \\
Hypertension & $8(15.4 \%)$ \\
Smoking & $5(9.6 \%)$ \\
Family history of moyamoya & $5(9.6 \%)$ \\
Suzuki stage (99 hemisphere) & \\
I & $3(3.0 \%)$ \\
II & $9(9.1 \%)$ \\
III & $27(27.3 \%)$ \\
IV & $52(52.5 \%)$ \\
V & $7(7.1 \%)$ \\
VI & $1(1.0 \%)$ \\
Location of hemorrhage & \\
Pure intraventricular & $22(42.3 \%)$ \\
Basal ganglia (+ventricle) & $2(3.8 \%)[+22(42.3 \%)]$ \\
Lobar hemorrhage & $5(9.6 \%)$ \\
Subarachnoid hemorrhage & $1(1.9 \%)$ \\
Usage of antiplatelet & $22(42.3 \%)$ \\
Perfusion status & \\
Decreased basal perfusion & $35(67.3 \%)$ \\
$\quad$ Mild & $8(15.4 \%)$ \\
$\quad$ Moderate & $14(26.9 \%)$ \\
Severe & $13(25.0 \%)$ \\
$\quad$ Mild & $34(65.4 \%)$ \\
Moderate & $15(28.8 \%)$ \\
Severe & $9(17.3 \%)$ \\
\hline & $10(19.2 \%)$ \\
\hline
\end{tabular}


median age at diagnosis was 36 (range, 21-60) years for all patients. Eight patients were male and $5(9.6 \%)$ had a family history of MMD. Nine patients (17.3\%) experienced TIA-like symptoms before the hemorrhagic event. Patient characteristics are detailed in Table 1.

The types of hemorrhage according to location were pure intraventricular hemorrhage (IVH, $n=22$ ), basal ganglia or periventricular hemorrhage with IVH $(n=22)$, lobar hemorrhage $(n=5)$, basal ganglia hemorrhage $(n=2)$, and subarachnoid hemorrhage $(n=1)$. Thirty-three patients $(63.5 \%)$ had transdural collaterals at initial evaluation. SPECT results showed that 44 (84.6\%) patients had impaired cerebral hemodynamics, 35 (67.3\%) had decreased basal perfusion, and 34 (65.4\%) had impaired vascular reserve. Twelve out of 52 hemorrhagic MMD patients were unable to determine the site of bleeding due to bilateral IVH which showed similar amount between each side of lateral ventricle. Other than these 12 patients, 31 out of 40 showed impaired cerebral hemodynamics on SPECT study. All of these patients showed ipsilateral perfusion abnormality.

\section{Treatment and clinical course}

Surgical treatment for the initial hemorrhagic event was performed in 19 (36.5\%) patients. Fourteen patients underwent external ventricular drainage for IVH, 3 underwent craniotomy hematoma removal, and 2 were treated with stereotactic aspiration and drainage.

Twenty-two patients (42.3\%) underwent direct or indirect bypass surgery due to a perfusion abnormality based on SPECT results. Among 22 surgically treated patients ( 32 hemisphere), 3 (3 hemisphere) underwent direct bypass surgery, and 19 (29 hemisphere) received indirect bypass surgery. Type of indirect bypass surgery was encephalo-duro-arterio-synangiosis (EDAS) using ipsilateral superficial temporal artery. Ten out of 22 underwent bilateral surgery based on the results of SPECT. The median time between the hemorrhagic event and revascularization procedure was 1 month (IQR, 1-7). Six patients who underwent direct and/or indirect bypass surgery received antiplatelet agents. And 18 patients without revascularization surgery received antiplatelets at physician's clinical discretion. Therefore, 22 patients (42.3\%) received either one or two antiplatelets during followup periods.

Seventeen patients experienced an ischemic $(n=5,9.6 \%)$ or hemorrhagic ( $n=12,23.1 \%$ ) event during the follow-up period (median 58 months, range 3-160). The estimated stroke-free survival rate was $5.8 \pm 1.4 \%$ /year (Fig. 1), while annual recurrent bleeding rate was estimated to be $4.9 \pm 1.3 \%$. Clinical outcomes after recurrent bleeding were as followed mRS $1=6(11.6 \%)$, mRS $2=1$ (1.9\%), mRS $3=1$ (1.9\%), mRS $4=2$ (3.9\%), mRS $5=1$ (1.9\%), and mRS $6=1(1.9 \%)$.

\section{Factors associated with recurrent bleeding}

Univariate analysis using the log-rank test showed that decreased basal perfusion $(p=0.017)$ was associated with recurrent

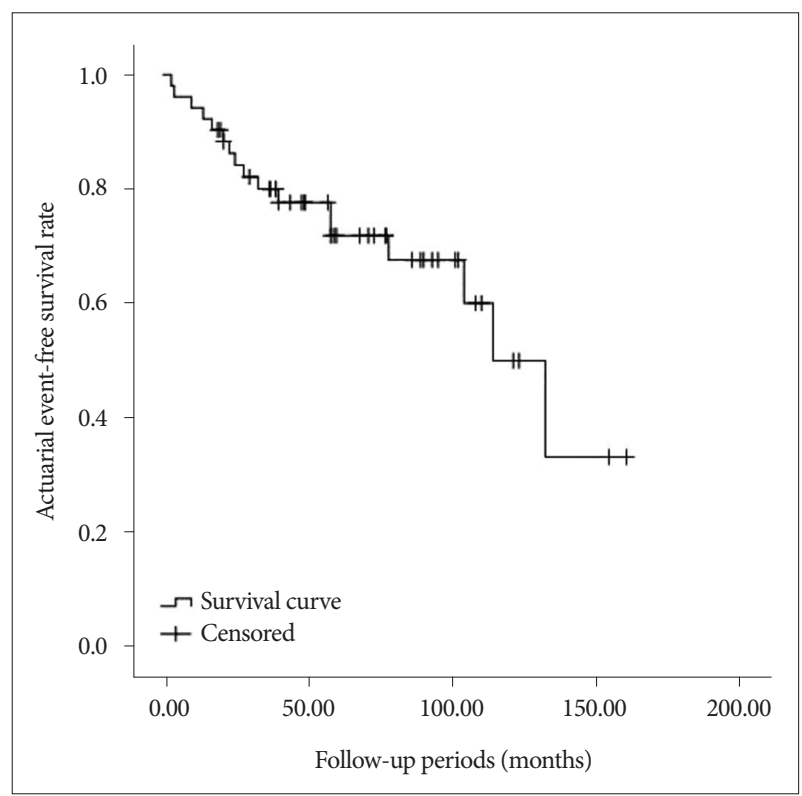

Fig. 1. Actuarial event-free survival rate in hemorrhagic moyamoya disease.

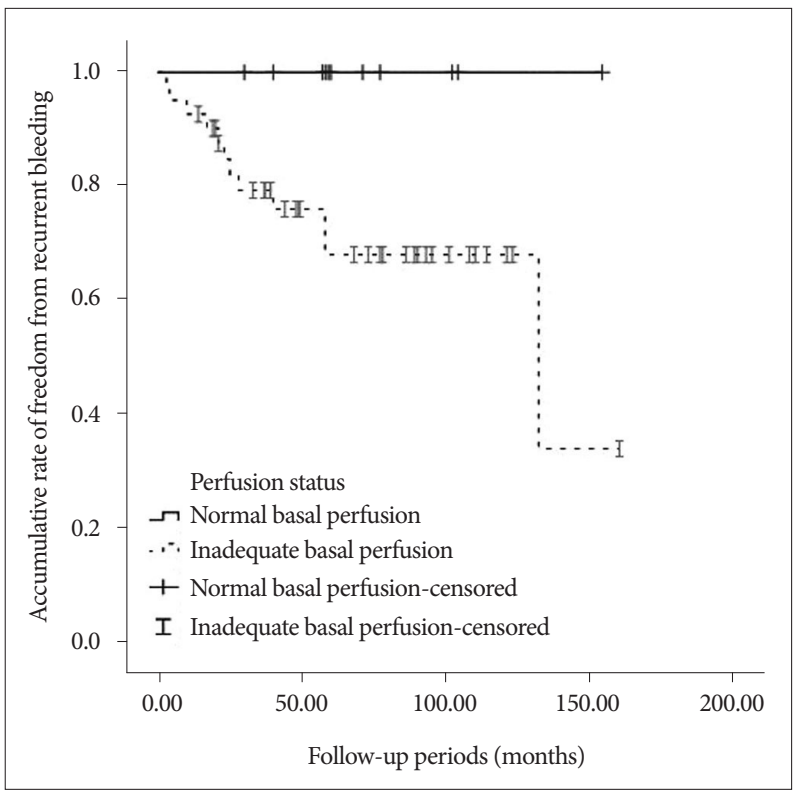

Fig. 2. Accumulative rate of freedom from recurrent hemorrhage via basal perfusion status in the single positron emission computed tomography analysis ( $p=0.017$, log-rank test).

bleeding (Fig. 2). Other variables did not show a significant association with recurrent bleeding; these variables included gender, age ( $\leq 40$ vs. $>40$ ), hypertension, smoking, impaired vascular reserve, and the use of an antiplatelet agent $(p>0.05)$.

Patients with decreased basal perfusion showed more recurrent stroke during the follow-up period ( $p=0.007,15 / 35$ vs. $2 / 17$ ). In addition, other variables did not show a statistically significant association with recurrent stroke.

No patient treated with direct bypass surgery showed hemorrhagic or ischemic events, and 6 of 19 treated with indirect by- 
pass surgery showed recurrent bleeding. No patients showed an ischemic event after revascularization. The log-rank test did not show any differences in a significant hemorrhagic event between surgically treated patients and patients without revascularization (Fig. 3). However, multivariate analysis using the Cox-regression method showed that decreased basal perfusion ( $p=0.037$, HR 19.87; 95\% CI=1.196-294.117) and no revascularization ( $p=0.016$, HR 10.218 ; 95\% CI=1.532-68.136) were associated with recurrent bleeding (Table 2).

\section{DISCUSSION}

A recent study demonstrated that surgical revascularization could reduce recurrent bleeding ${ }^{9,10)}$. Here, direct revascularization seemed to be more beneficial than indirect bypass surgery. Dilated anterior choroidal, posterior communicating artery, and moyamoya vessels are suspected as risk factors for a hemorrhagic event in MMD because these factors are thought to reflect the hemodynamic stress in that area ${ }^{11)}$. However, these aforemen-

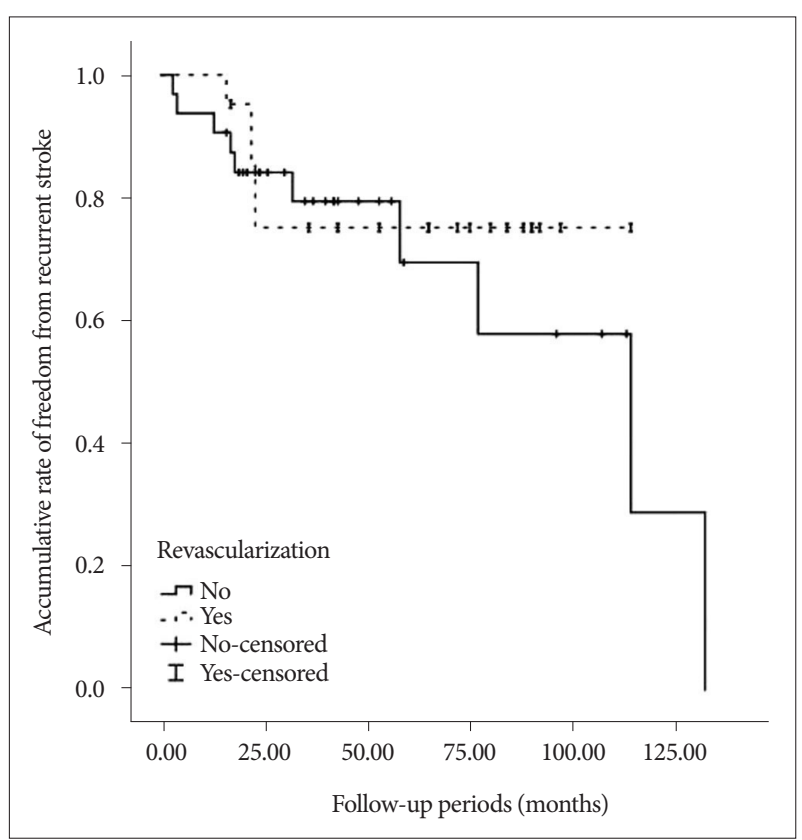

Fig. 3. Accumulative rate of freedom from recurrent stroke via the specific treatment methods ( $p=0.570$, log-rank test). tioned angiographic factors have no clear definition. Therefore, interpretation of angiographic findings can vary depending on the evaluator. The present study hypothesized that hemodynamic impairment could more precisely reflect hemodynamic stress. Theoretically, impaired perfusion results in hemodynamic stress on the vessel wall and facilitates dilation or microaneurysm formation in collateral vessels. Surgical treatment can reduce the development of abnormal collateral vessels by rectifying the hemodynamics. Considering this, surgical treatment seems to be helpful in $\mathrm{hMMD}^{10)}$. Although a recent prospective randomized trial demonstrated that direct bypass could lower rebleeding risk, the role of surgical treatment in hMMD is still controversial $^{2,5,9,15)}$. Kawaguchi et al. ${ }^{5)}$ reported that direct bypass is effective to prevent recurrent hemorrhage or ischemic events. However, Okada's long-term follow-up study found that despite significant improvement in cerebral hemodynamics and decreased abnormal collateral vessel development, around $20 \%$ of patients developed recurrent bleeding after surgical revascularization ${ }^{13)}$.

Our univariate analysis could not demonstrate fewer hemorrhagic events in the surgically treated group compared to the non-surgery group. Half $(n=6)$ of recurrent bleeding patients have received indirect revascularization. However, multivariate analysis suggested that surgery could reduce rebleeding risk. According to our strategy, surgical treatment was performed on patients with impaired hemodynamics. Since there was a lack of sufficient evidence regarding the preventive role of surgery on recurrent bleeding in hMMD, the main surgical goal was to prevent subsequent ischemic events among patients who were recovering without any significant neurologic deficits after the initial hemorrhagic event. According to our results, hemodynamic insufficiency seems to be an important predictor after initial bleeding. Based on the results of our revascularization strategy in hMMD, surgery was usually performed on patients with a high rebleeding risk. Therefore, the present results might reflect our treatment strategy. None of the patients treated with revascularization surgery experienced an ischemic event, while 5/30 nonsurgery patients had a recurrent TIA during follow-up. Additionally, a considerable proportion (17.6\%) of our hMMD patients had a history of ischemic events before the hemorrhage. Moreover, around $80 \%$ of our patients showed a hemodynamic abnormality. These findings suggest that the hemorrhagic subtype

Table 2. Multivariate analysis regarding recurrent bleeding

\begin{tabular}{|c|c|c|c|c|}
\hline \multirow{2}{*}{ Variable } & \multirow{2}{*}{$p$-value } & \multirow{2}{*}{ Hazard ratio } & \multicolumn{2}{|c|}{$95 \%$ confidence interval } \\
\hline & & & Lower & Upper \\
\hline Omission of revascularization & 0.016 & 10.218 & 1.532 & 68.136 \\
\hline Decreased basal perfusion & 0.037 & 19.872 & 1.196 & 294.117 \\
\hline Impaired vascular reserve & 0.114 & 4.762 & 0.669 & 33.325 \\
\hline Age $(<40)$ & 0.086 & 4.974 & 0.796 & 31.066 \\
\hline Gender (male) & 0.226 & 0.266 & 0.031 & 2.265 \\
\hline Hypertension & 0.787 & 1.437 & 0.104 & 19.921 \\
\hline Smoking & 0.187 & 0.150 & 0.009 & 2.521 \\
\hline Usage of antiplatelet & 0.224 & 2.569 & 0.562 & 11.742 \\
\hline
\end{tabular}


cannot be free from ischemic events, indicating that revascularization can provide beneficial effects in hMMD, even if only a marginally decreased risk of rebleeding is present. However, revascularization surgery should be applied with caution for selected hMMD patients with a hemodynamic abnormality.

Other concerns regarding disparities with previous studies could be related to the bleeding site. In MMD, the most common bleeding sites are deep in the brain, such as intraventricular sites or the basal ganglia, rather than at cortical and subcortical sites ${ }^{4,13}$. Among 12 patients with recurrent bleeding, half of the patients have received indirect revascularization. All the sites of recurrent bleeding were located in the deep side of the brain as described above. Furthermore bleeding sites were similar with the previous bleeding sites. Considering that direct or indirect bypass surgery usually provides additional blood flow to the fronto-temporo-parietal cortical and subcortical areas, surgery may have a limited role until revascularized vessels are able to influence deeper brain sites. Indirect revascularization might need a relatively extended time period in order to influence deeper brain site. Additionally, MMD is a rare disease and has a dynamic disease course, making it difficult to gather controlled data ${ }^{8,18)}$.

Several limitations should be noted in this study. First, this study only investigated half of the patients with hMMD in our institute. We did not perform a detailed examination on patients with poor clinical outcomes ( $m R S>4$ ) during recovery after the initial hemorrhagic event. And we excluded patients with early stage MMD and included those with unilateral (probable) MMD to reduce the diagnostic ambiguity. Therefore, a significant selection bias was likely present. It is possible that the subset of hMMD patients assessed just happened to be experiencing good recovery after the initial event. Nevertheless, these patients should be acknowledged since subsequent events occur in a high proportion of these patients, resulting in poor outcomes even after satisfactory recovery from the first hemorrhagic event ${ }^{7}$. Second, the present study used a qualitative analysis of previous hemodynamic studies, and follow-up SPECT and DSA were rarely used in our study population. This was the case even though such analyses could provide more detailed information regarding hemodynamic effects on hMMD. Finally, the present study was limited by a small sample size, relatively short follow-up periods, and retrospective analyses.

In conclusion, hemodynamic abnormalities seem to be associated with recurrent bleeding in MMD. Furthermore, ischemic events are common in hMMD, which result from hemodynamic insufficiency. To reduce the risk of further stroke in hMMD with hemodynamic insufficiency, revascularization should be considered.

\section{References}

1. Baba T, Houkin K, Kuroda S : Novel epidemiological features of moyamoya disease. J Neurol Neurosurg Psychiatry 79: 900-904, 2008

2. Gross BA, Du R : Adult moyamoya after revascularization. Acta Neuro- chir (Wien) $155: 247-254,2013$

3. Han JS, Abou-Hamden A, Mandell DM, Poublanc J, Crawley AP, Fisher JA, et al. : Impact of extracranial-intracranial bypass on cerebrovascular reactivity and clinical outcome in patients with symptomatic moyamoya vasculopathy. Stroke 42 : 3047-3054, 2011

4. Hwang K, Hwang G, Kwon OK : Endovascular embolization of a ruptured distal lenticulostriate artery aneurysm in patients with moyamoya disease. J Korean Neurosurg Soc 56 : 492-495, 2014

5. Kawaguchi S, Okuno S, Sakaki T : Effect of direct arterial bypass on the prevention of future stroke in patients with the hemorrhagic variety of moyamoya disease. J Neurosurg 93 : 397-401, 2000

6. Kikuta K, Takagi Y, Nozaki K, Sawamoto N, Fukuyama H, Hashimoto $\mathrm{N}$ : The presence of multiple microbleeds as a predictor of subsequent cerebral hemorrhage in patients with moyamoya disease. Neurosurgery 62: 104-111, discussion 111-112, 2008

7. Kobayashi E, Saeki N, Oishi H, Hirai S, Yamaura A : Long-term natural history of hemorrhagic moyamoya disease in 42 patients. J Neurosurg 93 : 976-980, 2000

8. Kuroda S, Hashimoto N, Yoshimoto T, Iwasaki Y; Research Committee on Moyamoya Disease in Japan : Radiological findings, clinical course, and outcome in asymptomatic moyamoya disease : results of multicenter survey in Japan. Stroke 38 : 1430-1435, 2007

9. Liu X, Zhang D, Shuo W, Zhao Y, Wang R, Zhao J : Long term outcome after conservative and surgical treatment of haemorrhagic moyamoya disease. J Neurol Neurosurg Psychiatry 84 : 258-265, 2013

10. Miyamoto S, Yoshimoto T, Hashimoto N, Okada Y, Tsuji I, Tominaga T, et al. : Effects of extracranial-intracranial bypass for patients with hemorrhagic moyamoya disease : results of the Japan Adult Moyamoya Trial. Stroke 45 : 1415-1421, 2014

11. Morioka M, Hamada J, Kawano T, Todaka T, Yano S, Kai Y, et al. : Angiographic dilatation and branch extension of the anterior choroidal and posterior communicating arteries are predictors of hemorrhage in adult moyamoya patients. Stroke 34 : 90-95, 2003

12. Nariai T, Matsushima Y, Imae S, Tanaka Y, Ishii K, Senda M, et al. : Severe haemodynamic stress in selected subtypes of patients with moyamoya disease : a positron emission tomography study. J Neurol Neurosurg Psychiatry 76:663-669, 2005

13. Okada Y, Shima T, Nishida M, Yamane K, Yamada T, Yamanaka C : Effectiveness of superficial temporal artery-middle cerebral artery anastomosis in adult moyamoya disease : cerebral hemodynamics and clinical course in ischemic and hemorrhagic varieties. Stroke 29:625-630, 1998

14. Ryan RW, Chowdhary A, Britz GW : Hemorrhage and risk of further hemorrhagic strokes following cerebral revascularization in Moyamoya disease : a review of the literature. Surg Neurol Int $3: 72,2012$

15. Saeki N, Nakazaki S, Kubota M, Yamaura A, Hoshi S, Sunada S, et al. : Hemorrhagic type moyamoya disease. Clin Neurol Neurosurg 99 Suppl 2 : S196-S201, 1997

16. Starke RM, Komotar RJ, Connolly ES : Optimal surgical treatment for moyamoya disease in adults : direct versus indirect bypass. Neurosurg Focus 26: E8, 2009

17. Suzuki J, Takaku A : Cerebrovascular "moyamoya" disease. Disease showing abnormal net-like vessels in base of brain. Arch Neurol 20 : 288-299, 1969

18. Yeon JY, Shin HJ, Kong DS, Seol HJ, Kim JS, Hong SC, et al. : The prediction of contralateral progression in children and adolescents with unilateral moyamoya disease. Stroke 42 : 2973-2976, 2011

19. Yoshida Y, Yoshimoto T, Shirane R, Sakurai Y : Clinical course, surgical management, and long-term outcome of moyamoya patients with rebleeding after an episode of intracerebral hemorrhage : an extensive follow-up study. Stroke 30 : 2272-2276, 1999 